\title{
Why health anxiety needs to be recognised in hospital practice
}

\author{
Author: Peter Tyrer ${ }^{\mathrm{A}}$
}

Health anxiety, formerly part of hypochondriasis, but now reformulated as excessive concern about health and, by extension, illness, comprises a large proportion of consultations in hospital practice. For too long it has been ignored in practice and not formally treated. This belief is no longer tenable, and in the last few years a number of easily administered psychological treatments have shown consistent benefit that help patients, practitioners and planners of services. A stepped care approach in which physicians and nurses are first helped to identify health anxiety, explain its significance to patients and then, if necessary, administer these treatments in the clinical setting without referral to psychiatric services, is recommended as a way forward. This approach should be embraced in secondary care.

KEYWORDS: Health anxiety, hypochondriasis, medically unexplained symptoms, psychological treatment, reassurance

DOI: $10.7861 /$ clinmed.2019-0346

\section{Introduction}

The average hospital physician sees five times more new psychiatric patients a week than does a clinical psychiatrist. This may be a surprise. A large proportion of people who consult physicians in hospital have unrecognised psychiatric problems, often combined with other medical disorders, but they are largely unrecognised. A proportion of these, probably a large proportion but at present good prevalence data are few, are represented by health anxiety (illness anxiety in the USA), the rest being reclassified recently in the American classification, Diagnostic and statistical manual of mental disorders (DSM-5), as 'somatic symptom disorder'. In the forthcoming International Classification of Diseases (ICD-11) classification, to be introduced in 2022, health anxiety is subsumed under the diagnosis of hypochondriasis as a 'residual category' and so, unfortunately, is not yet accepted fully as a diagnosis. But, however it is named, it is common and, in one recent study, one in five of all patients attending medical outpatients had the condition. ${ }^{2}$ This compares with other studies in which the proportion of all symptoms lacking a medical

Author: ${ }^{A}$ emeritus professor of community psychiatry, Imperial College London, London, UK explanation is nearer to $50 \%{ }^{3}$ The average psychiatrist sees two to four new patients a week, so will never approach the numbers seen in medical care.

Thus, we have a condition that is firmly embedded in secondary care at all levels but which is currently not part of medical intervention. This requires urgent review.

\section{How are patients with health anxiety currently treated in hospital clinics?}

There has been little change in management of health anxiety until comparatively recently. Hypochondriasis was a diagnosis to be aware of but not to treat, as the general view from both the mental health and medical viewpoint was that people had to live with their worries, thought to be intrinsic to personality and not amenable to intervention. ${ }^{4}$ Physicians have had intensive training in the conditions for which they have the expertise to treat, but this does not include those that commonly present if they are primarily related to mental health. As a consequence, the interpretation of these symptoms by physicians is uneven and often unhelpful, and the there is a tendency to ignore, or at least minimise, the psychological element when making diagnoses., Because training and understanding is lacking, there is an understandable tendency to reassure and thereby detract from the severity of the psychological condition and also to expect that a set of tests showing absence of pathology will satisfy the patient. These actions are usually counter-productive as they reinforce the abnormal health beliefs. ${ }^{7,8}$ This is because supportive, well-meaning actions, though helpful to most people, are interpreted very differently by the health anxious patient (Table 1).

The central elements of health anxiety are undue worry about health and the fear of developing a disease. Commonly, the symptoms of worry and anxiety, which include many bodily symptoms, are misinterpreted as evidence of organic illness. Autonomic and somatic symptomatology, such as flushing, palpitations, dizziness, difficulty in breathing, tremor, skin marks and blemishes, protrusions and bumps, twitching of muscles, and pains and aches, are all given sinister significance and serious illness needs to be excluded. Each sufferer becomes their own monitor of health but, as they are never convinced that they are doing the job properly, they also need attention from medical personnel to confirm their fears. Once established, health anxiety leads to continued vigilance, often associated with checking of the body and internet browsing (cyberchondria), followed by the vicious cycle of increased anxiety, greater symptomatology and more misinterpretation. 
Table 1. The negative interpretation of reassurance by the health anxious patient

\section{Common explanations by physicians}

'All the tests are normal there is nothing to worry about.'

'There is really nothing wrong with you that we can detect.'

'I can reassure you that all is well.'

'I think it might be best for you to see a psychologist.'

\section{Interpretation by health anxious} patient

'I still have the symptoms - they must have done the wrong tests.'

'I must be in the wrong clinic - I will ask my general practitioner to make a new referral.'

'I felt better at first, but the symptoms have come back again the doctor must be wrong.'

'They think this problem is all in my mind - the last thing I will agree to is a mental health referral.'

It is this cycle that needs to be understood in medical settings, and also explains why very few of the patients ever appear in psychiatric settings; they are convinced their problems are medical.

\section{Can health anxiety be treated successfully?}

In the last 20 years attitudes have changed greatly about health anxiety, particularly with regard to psychological treatments, with most evidence obtained with forms of cognitive behavioural therapy (CBT) but also including stress management, mindfulness and acceptance, and commitment therapy. ${ }^{10-12}$ There is also support for treatment with selective serotonin reuptake inhibitors but trials have generally shown fairly high levels of drop-out. ${ }^{13,14}$ One of the big advantages for health services following successful treatment is a reduction in medical attendances and consequent cost savings. ${ }^{15,16}$ The duration of benefit with successful treatment is also very long, for at least 5 years, and far superior to standard care. ${ }^{17}$

The common reaction by physicians to information of this nature is to note briefly and then think of referral to the nearest liaison psychiatry team. But this is too big a problem for liaison psychiatry. If a cardiologist sees 20 patients with health anxiety in the course of an average week there is no possibility of a feasible referral process to an external team.

The problem of health anxiety should be tackled in-house. It already has been introduced in some services. So, for example, John Boyd Chambers and his group at St Thomas' Hospital in London have introduced a stepped-care intervention for non-cardiac chest pain in a team with an attached clinical psychologist. ${ }^{18}$ The three components of this are:

> simple explanation and formulation of the factors affecting pain and reassurance (which can be given by anybody in the team)

> low-intensity CBT for straightforward cases including relaxation and breathing techniques (given by a trained nurse)

> high-intensity CBT for cases with complex psychosocial issues, high levels of anxiety or depression, or who do not respond to low-intensity CBT (given by the psychologist).

In our own work we have found that a clinical psychologist may not be necessary except as a supervisor or trainer. This is because general nurses with no special knowledge of psychological treatments have been shown to be excellent therapists once they have been trained in psychological techniques. They may even be superior to psychologists in that they have more credibility with those patients who are averse to mental health professionals. ${ }^{19}$

One of the most important parts of the stepped-care programme is the initial one. You have to detect health anxiety before any form of management can be introduced. One of the central arguments in this review is to persuade every physician that this is one of their essential tasks. If they do not carry it out, nobody else will do it for them. It is only necessary to ask a few simple questions. ${ }^{8}$

$>$ Do you worry a lot about your health?

$>$ What is the worst thing you fear that could happen?

$>$ When did you start to worry more about your health?

> How has this worry about your health affected your work/social/ family life?

These are all easy to introduce into a clinical interview, and need to be asked when a clinical problem does not seem to be solved or further conventional medical investigations appear to be pointless. One of the important triggers for health anxiety is a previous physical illness. In the past, the diagnosis of hypochondriasis (the precursor to health anxiety) was only considered if physical illness was absent but, to take an example, if you have a myocardial infarction out of the blue at the age of 45 years, even if it is followed by a highly successful insertion of a stent, it is not uncommon for a patient to become highly concerned about an episode of chest pain, a fluttering of the heart or unexpected shortness of breath, even when these have no connection to physical illness.

Telling patients that they have, or are very likely to have, health anxiety is not a task that should be left to junior staff or nurses. Treatment, after identification, can be carried out by others but the health anxious patient hangs on every word of the senior doctor. If others take on the responsibility it may deflect questions for a time but eventually it will have to be repeated by a senior colleague.

\section{Is health anxiety more of a problem in some clinics than others?}

People worry more about their health when their symptoms are not easily visible or cannot be simply explained or resolved by common tests. So, in our research in secondary care, we have found that the highest levels of anxiety are found in patients in neurology, gastroenterology and cardiology clinics and the least in genitourinary medicine. ${ }^{15,17}$

The population is now being asked to take more responsibility for monitoring their own health. This is not unreasonable, but it puts a particular strain on those with health anxiety, as they are the ultimate health monitors, often checking many times a day on their pulse rates, their external orifices and what comes out of them, and anything else that can be conveniently measured. We have even had a patient who measured his abdominal girth every day because he was convinced his body was ripe for an explosion. The need to take action over warning signs is most prevalent for cardiac symptoms. When NHS information sites advise you to 'call 999 for an ambulance immediately if you develop sudden severe chest pain', patients with excessive health anxiety set a very low bar for such a call and also attend emergency departments frequently, but very few have any significant medical pathology. ${ }^{20,21}$ What is encouraging is that these patients appear to respond very well to psychological intervention, provided it is offered in an appropriate way. ${ }^{16,22,23}$ 


\section{Are people with health anxiety 'the worried well'?}

There is a view, more prevalent in some places than others, that the people with health anxiety and similar symptoms have nothing seriously wrong with them and are just serial complainers. They are often given the collective title of 'the worried well'. This is a misjudged expression. Those with health anxiety and associated disorders suffer greatly but usually silently. A proportion of them never seek advice at all, fearing that they have a serious illness but not having the nerve to put it to the test. The well-known film director, Tony Scott, jumped to his death from a bridge in Los Angeles in August 2012 believing he had inoperable brain cancer, but nothing was found on autopsy. ${ }^{24}$ Like many who have health anxiety, Scott had received treatment for cancer in the past but there was no sign of recurrence. He had not consulted a doctor in the recent past.

Longitudinal studies have shown that people with health anxiety (and other forms of anxiety) die prematurely and also tend to get significantly depressed if untreated; those who have had psychological treatment do not become depressed. 16,25,26

\section{The way forward}

There is a term that can easily be dismissed as jargon but should not be: mental health literacy. When such literacy is high, mental illness, in all its forms, is identified readily and, whenever possible, managed appropriately. Liaison psychiatry can help to improve mental health literacy but, because health anxiety is so common, such services cannot do this on their own. Most physicians probably need to improve awareness of mental illness; it is easy to suggest this but difficult to implement when pressures in their disciplines are so high. But even a small change can create great benefit, especially if other staff are similarly literate and can identify the relevant symptoms early. It is possible to envisage that specialist ancillary staff in many medical clinics can be trained in the essentials of recognising and treating health anxiety and to devote one or two of their clinical sessions to this work. This would not only relieve the suffering of many patients but also reduce unnecessary consultation, laboratory and radiology staff workload, and the irritable contretemps of people who tend to request second opinions and unnecessary tests. Whatever transpires, the present pattern of unhelpful reassurance should not continue.

\section{References}

1 American Psychiatric Association. Diagnostic and statistical manual of mental disorders, 5th edn. Arlington: American Psychiatric Association, 2013:215.

2 Tyrer P, Cooper S, Crawford M et al. Prevalence of health anxiety problems in medical clinics. J Psychosom Res 2011;71:392-4.

3 Nimnuan C, Hotopf M, Wessely S. Medically unexplained symptoms: an epidemiological study in seven specialities. J Psychosom Res 2001;51:361-7.

4 Adler G. The physician and the hypochondriacal patient. N Engl J Med 1981;304:1394-6.

5 Warner A, Walters K, Lamahewa K, Buszewicz M. How do hospital doctors manage patients with medically unexplained symptoms: a qualitative study of physicians. J R Soc Med 2017;110:65-72.

6 Poloni N, Ielmini M, Caselli I et al. Medically unexplained symptoms in hospitalized patients: a 9-year retrospective observational study. Front Psychiatry 2018;9:626.

7 Salkovskis PM, Warwick HM. Morbid preoccupations, health anxiety and reassurance: a cognitive-behavioural approach to hypochondriasis. Behav Res Ther 1986;24:597-602.
8 Hedman-Lagerlöf E, Tyrer P, Hague J et al. Health anxiety. BMJ 2019;364:1774.

9 Te Poel F, Baumgartner SE, Hartmann T et al. The curious case of cyberchondria: A longitudinal study on the reciprocal relationship between health anxiety and online health information seeking. J Anxiety Disord 2016;43:32-40.

10 Olatunji BO, Kauffman BY, Meltzer S et al. Cognitive-behavioral therapy for hypochondriasis/health anxiety: a meta-analysis of treatment outcome and moderators. Behav Res Ther 2014;58:65-74.

11 Eilenberg T, Fink P, Jensen JS, Rief W, Frostholm L. Acceptance and commitment group therapy (ACT-G) for health anxiety: a randomized controlled trial. Psychol Med 2016;46:103-15.

12 Tyrer P. Recent advances in the understanding and treatment of health anxiety. Curr Psychiat Rep 2018;20:49.

13 Greeven A, van Balkom AJLM, Visser $S$ et al. Cognitive behavior therapy and paroxetine in the treatment of hypochondriasis: a randomized controlled trial. Am J Psychiatry 2007:164:91-9.

14 Fallon BA, Ahern DK, Pavlicova M et al. A randomized controlled trial of medication and cognitive-behavioral therapy for hypochondriasis. Am J Psychiatry 2017;174:756-64.

15 Seivewright H, Salkovskis P, Green J et al. Prevalence and service implications of health anxiety in genitourinary medicine clinics. Int J STD AIDS 2004;15:519-22.

16 Morriss R, Patel S, Malins S et al. Clinical and economic outcomes of remotely delivered cognitive behaviour therapy versus treatment as usual for repeat unscheduled care users with severe health anxiety: a multicentre randomised controlled trial. BMC Medicine 2019;17:16.

17 Tyrer P, Salkovskis P, Tyrer $\mathrm{H}$ et al. Cognitive behaviour therapy for health anxiety in medical patients (CHAMP): randomised controlled trial with outcomes to five years. Health Technol Assess 2017;21:50.

18 Chambers JB, Marks EM, Hunter MS. The head says yes but the heart says no: what is non-cardiac chest pain and how is it managed? Heart 2015;101:1240-9.

19 Tyrer $\mathrm{H}$, Tyrer $\mathrm{P}$, Lisseman-Stones $\mathrm{Y}$ et al. Therapist differences in a randomised trial of the outcome of cognitive behaviour therapy for health anxiety in medical patients. Int J Nurs Stud 2015;52:686-94.

20 NHS Inform. Chest pain. NHS, 2020. www.nhsinform.scot/illnessesand-conditions/heart-and-blood-vessels/conditions/chest-pain

21 Sekhri N, Feder GS, Junghans C, Hemingway H, Timmis AD. How effective are rapid access chest pain clinics? Prognosis of incident angina and non-cardiac chest pain in 8762 consecutive patients. Heart 2007;93:458-63.

22 Mayou RA, Bryant BM, Sanders D et al. A controlled trial of cognitive behavioural therapy for non-cardiac chest pain. Psychol Med 1997;27:1021-31.

23 Tyrer P, Tyrer H, Morriss R et al. Clinical and cost-effectiveness of adapted cognitive behaviour therapy for non-cardiac chest pain: multicentre, randomised controlled trial. Open Heart 2017:4:e000582.

24 Miller ]. Tony Scott suicide remains a mystery after autopsy. HWD Daily 2012.

25 Tolmunen T, Lehto SM, Julkunen J, Hintikka J, Kauhanen J. Trait anxiety and somatic concerns associate with increased mortality risk: a 23-year follow-up in aging men. Ann Epidemiol 2014;24:463-8.

26 Kikkenborg Berg S, Caspar Thygesen L, Hastrup Svendsen ] et al. Anxiety predicts mortality in ICD patients: results from the crosssectional national CopenHeartICD survey with register follow-up. Pacing Clin Electrophysiol 2014;37:1641-50.

Address for correspondence: Prof Peter Tyrer, Imperial College London, Centre for Psychiatry, 7th Floor, Commonwealth Building, Hammersmith Hospital, London W12 0NN, UK. Email: p.tyrer@imperial.ac.uk 


\section{Working flexibly: a toolkit}

This new resource discusses opportunities for physicians to work flexibly as an alternative to leaving the NHS and provides examples of flexible ways of working.

The toolkit - available as a download and an online resourcewill allow you to discover 'tried and tested' ways of working flexibly and top tips for all stages of your career.

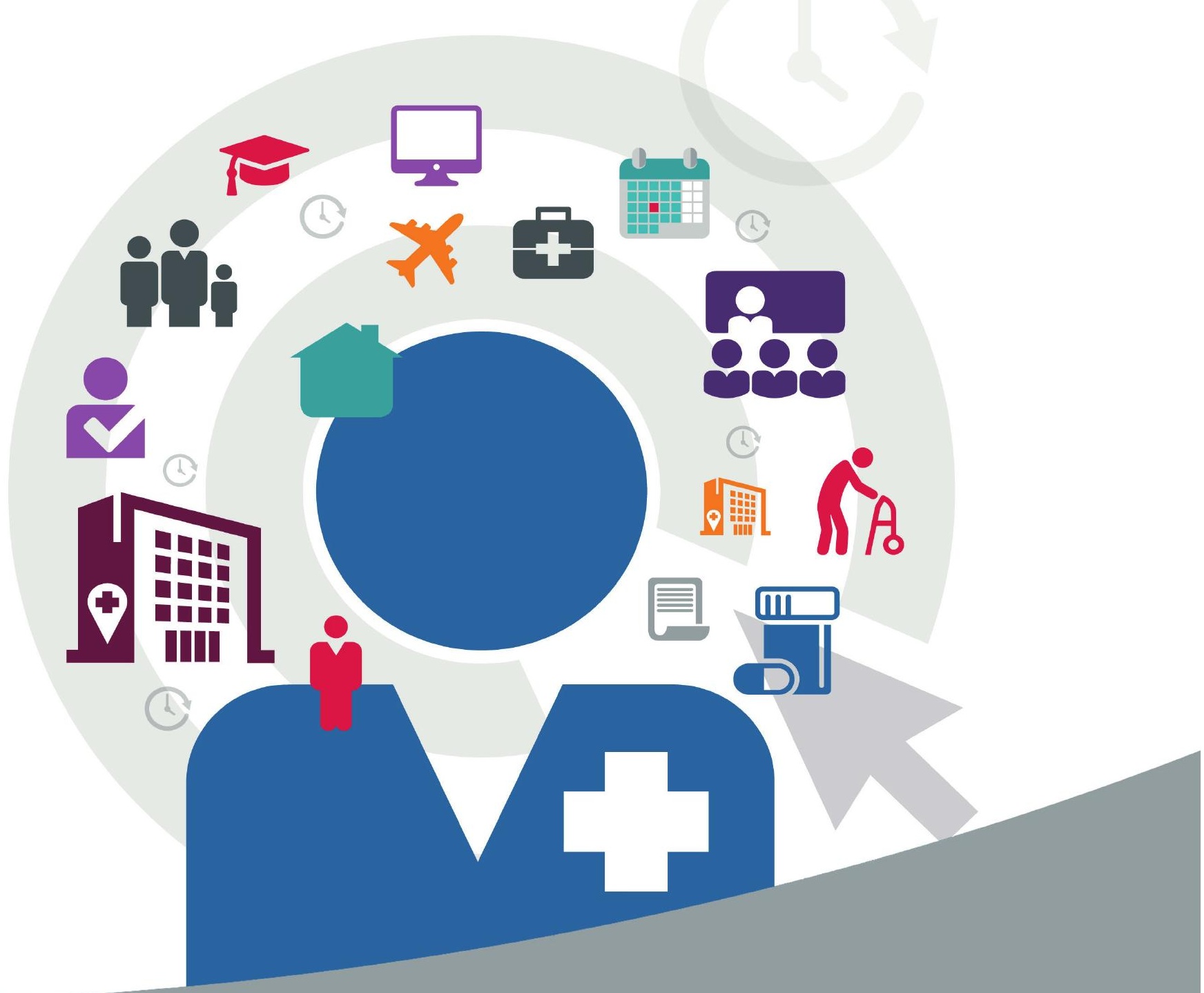

